\title{
Analysis of a WDM Packet Switch with Improved Performance under Bursty Traffic Conditions due to Tuneable Wavelength Converters
}

Danielsen, Søren Lykke; Jørgensen, Carsten; Mikkelsen, Benny; Stubkjær, Kristian

Published in:

Journal of Lightwave Technology

Link to article, DOI:

$10.1109 / 50.669000$

Publication date:

1998

Document Version

Publisher's PDF, also known as Version of record

Link back to DTU Orbit

Citation (APA):

Danielsen, S. L., Jørgensen, C., Mikkelsen, B., \& Stubkjær, K. (1998). Analysis of a WDM Packet Switch with Improved Performance under Bursty Traffic Conditions due to Tuneable Wavelength Converters. Journal of Lightwave Technology, 16(5), 729-735. https://doi.org/10.1109/50.669000

\section{General rights}

Copyright and moral rights for the publications made accessible in the public portal are retained by the authors and/or other copyright owners and it is a condition of accessing publications that users recognise and abide by the legal requirements associated with these rights.

- Users may download and print one copy of any publication from the public portal for the purpose of private study or research.

- You may not further distribute the material or use it for any profit-making activity or commercial gain

- You may freely distribute the URL identifying the publication in the public portal 


\title{
Analysis of a WDM Packet Switch with Improved Performance Under Bursty Traffic Conditions Due to Tuneable Wavelength Converters
}

\author{
Soren L. Danielsen, Carsten Joergensen, Benny Mikkelsen, and Kristian E. Stubkjaer, Member, IEEE
}

\begin{abstract}
For realistic traffic, i.e., bursty traffic patterns, the use of tuneable wavelength converters is recognized as essential for reducing the complexity of photonic wavelength division multiplexing (WDM) packet switches. Results are obtained from an analytical traffic model that includes buffering in the wavelength domain and accounts for bursty traffic. The theoretical model is verified by simulations and from the model we find that higher traffic loads as well as burstiness can be accepted when tuneable wavelength converters are used. Consequently, a larger throughput of the photonic packet switches is obtained and very importantly, this is achieved while keeping the number of gates needed to realize the space switches nearly constant.
\end{abstract}

Index Terms - Optical buffering, optical networks, optical packet switching, traffic analysis, wavelength conversion.

\section{INTRODUCTION}

$\mathbf{T}$ HE implementation of future transparent optical networks is likely to use wavelength division multiplexing (WDM) to obtain high flexibility and network throughput [1]-[3]. Moreover, better use of the bandwidth is attained by implementing packet switched network layers [4]. Consequently, the study of optical WDM packet switches is of considerable interest [5]-[7]. The buffers in such switches can be realized with fiber delay-lines [6], [7]. However, the complexity of the switch increases with the number of fiber delay-lines, so a reduction of the fiber buffers is preferable. This is especially true under bursty traffic conditions where the required buffer capacity increases significantly compared to uniformly distributed traffic [8]. Here, we present an analytical traffic model for bursty traffic that is a generalization of the model presented in [9]. The presented model is also extended to account for the use of the wavelength domain to avoid contention of optical packets at the switch outlet. By using tuneable optical wavelength converters (TOWC's) to assign packets to unused

Manuscript received June 10, 1997; revised February 4, 1998. This work was supported in part by research program ACTS AC043, KEOPS.

S. L. Danielsen and K. E. Stubkjaer are with the Technical University of Denmark, Department of Electromagnetic Systems, Center for Broadband Telecommunications, Lyngby DK-2800 Denmark.

C. Joergensen was with the Technical University of Denmark, Department of Electromagnetic Systems, Center for Broadband Telecommunications, Lyngby DK-2800 Denmark. He is now with Ericsson, Denmark.

B. Mikkelsen was with the Technical University of Denmark, Department of Electromagnetic Systems, Center for Broadband Telecommunications, Lyngby DK-2800 Denmark. He is now with Lucent Technologies, Holmdel, NJ 07733 USA.

Publisher Item Identifier S 0733-8724(98)03316-7.

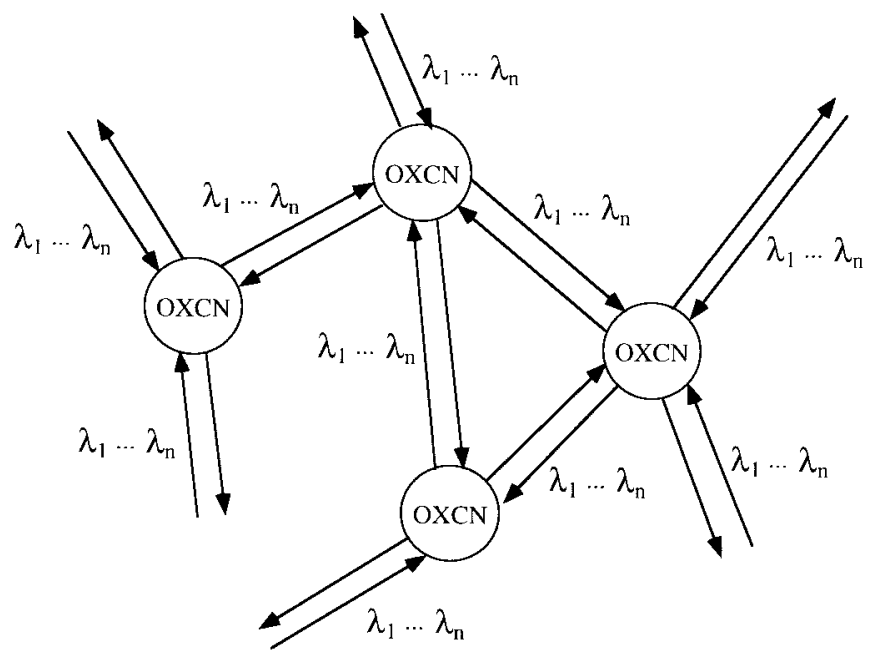

Fig. 1. General WDM network where $n$ wavelength channels are transmitted between each switching node.

wavelengths in the fiber delay-line buffers we show that a reduction of the number of fiber delay-lines in the WDM packet switch is obtained. Consequently, the implementation of large buffers usually needed under bursty traffic conditions is avoided.

Our investigation is carried out as follows. First we derive (Section II) and second verify (Section III) an analytical traffic model that describes the performance of the optical packet switch with fiber delay-line output buffers. In Section IV, the model is then used to analyze the traffic performance of the switch focusing on packet loss probabilities as well as possible switch throughputs when the burstiness of the traffic changes. Additionally, we consider the number of wavelength channels needed for a desired throughput. The investigation compares the performance with and without TOWC's.

\section{SWITCH AND NeTwork DESCRIPTION}

The general optical WDM mesh network considered is shown in Fig. 1 where $n$ different wavelengths, $\lambda_{1} \cdots \lambda_{n}$, are used to carry packet traffic between the optical network nodes. The nodes may be realized as seen in Fig. 2 for a $N \times N$ switch ( $N$ input and output fibers). The switch consists of three main blocks: 1) a demultiplexer (DMUX) per input fiber that selects the packets arriving at the $n$ fixed wavelengths, $\lambda_{1} \cdots \lambda_{n}$, followed by TOWC's that address free 


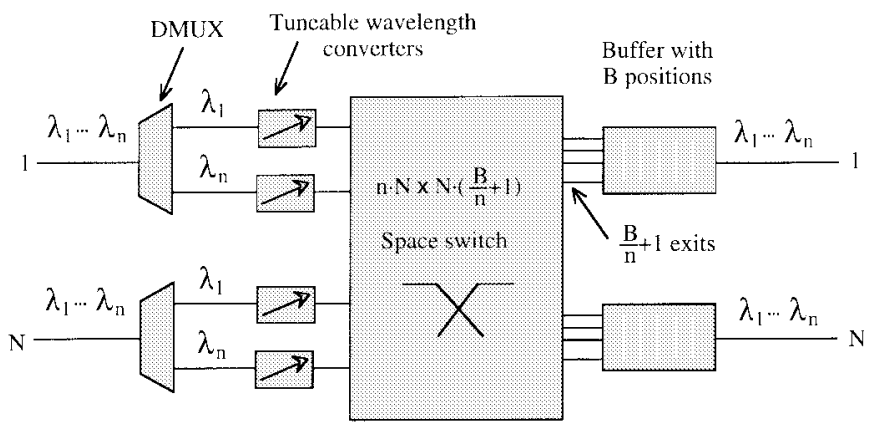

Fig. 2. WDM packet switch with buffers realized as fiber delay-lines and with tuneable wavelength converters to address free space in the buffers. $N$ is the number of in- and outlets, $B$ the number of cell positions in the buffer while $n$ is the number of wavelength channels per in- and outlet.

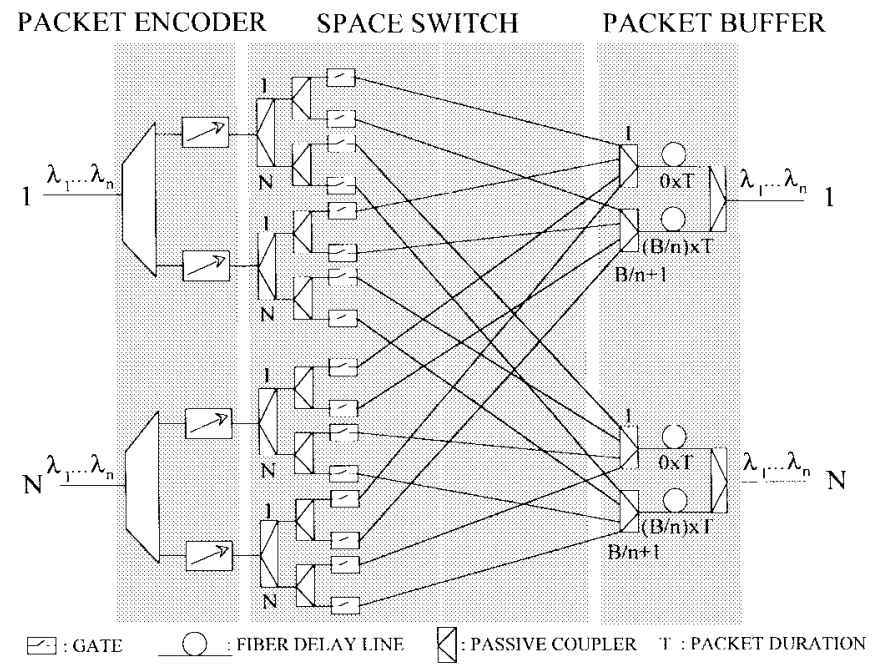

Fig. 3. WDM packet switch with gates to form a space switch and fiber delay lines to realize optical buffers.

space in the fiber delay-line output buffers, 2) a space-switch to access the buffers, and 3) packet buffers, that are realized by fiber delay-lines. Not shown in the architecture are optical to electrical interfaces situated just after the demultiplexers at the switch inlets as in [7]. These interfaces are used to extract the packet header information that is used to route the packet to the correct switch outlets. With this information as well as knowledge of the queueing situation, the output wavelength of the converters as well as the state of the gates within the space switch can be electrically controlled. Furthermore synchronous arrival of packets is assumed and could be realized as shown in, e.g., [10] and [11]. A more detailed view of the switch architecture is shown in Fig. 3 where the space switch is build with $N \times n N \cdot(B / n+1)$ gates and where the buffers consisting of fiber delay-lines are illustrated together with a connection without delay, $0 \times T . N$ is the number of in- and outlets while $B$ is the number of packet positions in the buffer, $n$ the number of wavelengths, and hence $B / n$ the number of fiber delay lines.

\section{TRAFFiC Model FOR Bursty TRAFFiC}

In this section, we derive a traffic model of the optical packet switch. It is a generalization of the model for bursty traffic developed in [9] where single channel in- and outlets

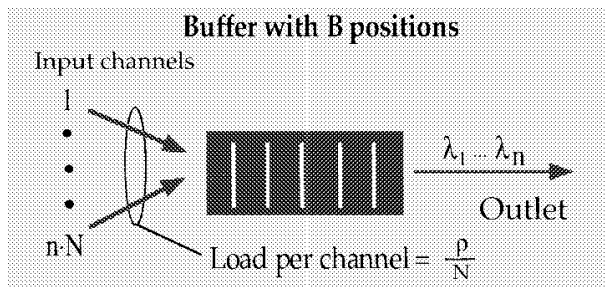

Fig. 4. Arrival process to the queue: $n \cdot N$ traffic sources of load $\rho / N$ all address the given queue leading to a total of $n \cdot \rho$ packets in mean going to the queue in each time slot (time slot $=$ packet period).

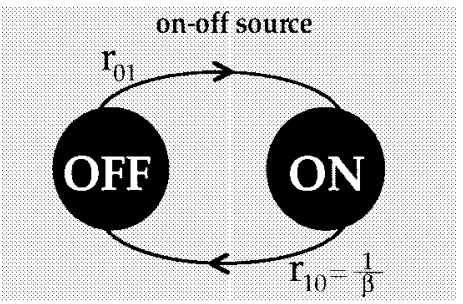

Fig. 5. Schematic of the model for the on-off sources. $r_{01}$ is the transition probability for going from off-state to on-state while $r_{10}$ is the probability for going to the off-state when initially in the on-state. The mean burst length, $\beta$, is given as $1 / r_{10}$.

were considered. Our modifications take into account the use of the wavelength dimension for buffering. First, we model the traffic sources to have bursty nature. Next, state transition probabilities for the number of active sources addressing a given tagged outlet are found. Following this, we derive a set of linear equations linking the packet arrivals with the distribution of packets in the queue. This in turn allows calculation of the packet loss probability.

\section{A. Traffic Source Modeling}

As seen from Figs. 2 and 3 a total of $n \cdot N$ channels enter the switch block. In our model we assume equal probabilities for the packets of these channels to go to any of the $N$ outlets. Therefore, if the load per channel is, $\rho$, each channel generates a load of $\rho / N$ to each of the $N$ output queues. The actual selection of a given outlet is not included in the model and the arrivals to a tagged output buffer are therefore modeled as $n \cdot N$ independent contributions each with a load of $\rho / N$ where $n \cdot N$ is the total number of channels going into the switch (see Fig. 4). Please note, that correlation between traffic on various wavelength channels is neglected. It must be pointed out, that in case traffic from the same source is split into several wavelength channels this assumption is not valid. However, since we are assuming that traffic on different channels origin from different sources correlation is not an issue here.

To include the bursty nature of the traffic sources we model these as on-off sources [12] as depicted in Fig. 5. In the figure, $r_{01}$, denotes the probability of a source going from the idle (off) to the active (on) state while $r_{10}$ denotes the reverse process. Clearly, the mean number of time intervals (time interval $=$ packet period) that the source stays in the on-state is given as $1 / r_{10}=\beta$ where $\beta$ is the mean burst length or burstiness. As in [12], [13] the idle and active periods of each of the identical $n \cdot N$ sources are assumed to be geometrically distributed with means of $1 / r_{01}$, and $\beta$, respectively. The 


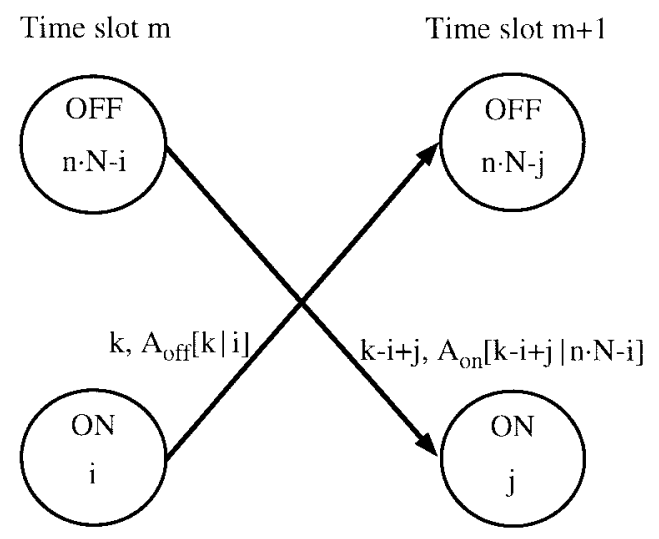

Fig. 6. State transitions going from time slot $m$ to time slot $m+1$. In time slot $m, i$ sources are on out of $n \cdot N$ while in time slot $m+1, j$ sources are on. $A_{\text {off }}$ signifies the probability of a source going from the on to the off state while $A_{\text {on }}$ gives the reverse probability.

probability for a source to be active is given as:

$$
P[\text { source is active }]=\frac{r_{01}}{r_{01}+r_{10}} .
$$

Furthermore, due to the convention given in Fig. 4 where one $N$ th of the traffic is destined for the considered outlet, this probability has to be equal to $\rho / N$. Therefore, when the mean burst length, $\beta$, and channel load, $\rho$, as well as switch size have been determined, $r_{01}$ can be calculated

$$
\frac{\rho}{N}=\frac{r_{01}}{r_{01}+r_{10}} \Leftrightarrow r_{01}=\frac{\frac{\rho}{N} r_{10}}{1-\frac{\rho}{N}}=\frac{\frac{\rho}{N} \frac{1}{\beta}}{1-\frac{\rho}{N}} .
$$

Having modeled the traffic sources we turn to the description of the number of sources that are active in each time slot.

\section{B. State Transition Probabilities for the Number of Active Sources}

In a given time slot, $m$, it is assumed that $i$ sources are in the active state. Assuming this, we derive the probability, $A_{i, j}$, for having $j$ active sources (among the total of $n \cdot N$ that can address the tagged output buffer) in the next time slot, $m+1$ (see Fig. 6)

Given there are $i$ sources in the on-state, the probability that $k$ of these on-state sources are turned off is expressed by, $A_{\text {off }}[k \mid i]$. The probability of having $j$ sources in the on-state in the next time slot is thus found by multiplying $A_{\text {off }}[k \mid i]$ by $A_{\text {on }}[j-i+k \mid n \cdot N-i]$, where the latter denotes the probability of $j-i+k$ sources becoming active among the $n \cdot N-i$ sources initially being in the off-state. In conclusion, we can write $A_{i, j}$ as

$$
\begin{aligned}
A_{i, j}= & \sum_{k=\max (0, i-j)}^{\min (i, n \cdot N-j)} A_{\mathrm{off}}[k \mid i] \cdot A_{\mathrm{on}}[k-i+j \mid n \cdot N-i] \\
= & \sum_{k=\min (0, i-j)}^{\min (i, n-j)}\left[\left(\begin{array}{l}
i \\
k
\end{array}\right) r_{10}^{k}\left(1-r_{10}\right)^{i-k}\right] \\
& \cdot\left[\left(\begin{array}{l}
n \cdot N-i \\
k-i+j
\end{array}\right) r_{01}^{k-i+j}\left(1-r_{01}\right)^{n \cdot N-k-j}\right]
\end{aligned}
$$

where the borders in the summation take boundary conditions into account. The above equation signifies the arrival process to each queue in the switch and thereby the state transitions describing the interaction of the arrivals and the number of packets in the queue can be found as shown in the following.

\section{Linear Equations to Find the Joint Probabilities for Packet Arrivals and Number of Packets in the Queue}

In order to fully describe the combination of the queuing and arrival processes we derive linear equations to describe the joint probability, $\pi_{i, j}$, for having $i$ packets in the queue at the end of a time slot and $j$ packets arriving at the beginning of the following time slot.

As the basis for writing these equations, the formula describing the number of packets in the queue at the end of two consecutive time slots $Q_{m}$ and $Q_{m+1}$ is used

$$
Q_{m+1}=\min \left[B, \max \left(0, Q_{m}+D_{m+1}-n\right)\right]
$$

where $D_{m+1}$ is the number of packets arriving at the beginning of time slot $m+1$.

Using (4) and assuming stationary conditions, we see that for $i=0$ and $j=0, \cdots, n \cdot N, \pi_{i, j}$ can be written as

$$
\pi_{0, j}=\sum_{k=0}^{k=n} \sum_{l=0}^{n-k} \pi_{k, l} \cdot A_{l, j} .
$$

The equation illustrates that there are $k$ packets in the queue, $0 \leq k \leq n$ and $l(l \leq n-k)$ new packets arrive, $\pi_{k, l}$. All $k+l \leq n$ packets are then removed immediately leaving an empty queue, $i=0$. Following this, $j$ packets arrive, $A_{l, j}$.

The principle of writing (5) is now repeated for all combinations of $i$ and $j$. In the case where $i \neq 0$ but $i<B$ while $j=0, \cdots, n \cdot N$ we get

$$
\pi_{i, j}=\sum_{k=\max (0, B-i-n)}^{\min (n \cdot N, i+n)} \pi_{i+n-k, k} \cdot A_{k, j} .
$$

The equation illustrates that there are $i+n-k$ packets in the queue and $k$ new packets arrive, $\pi_{i+n-k, k}$. Following this, $n$ packets are removed leaving a queue with $i$ packets. Finally, $j$ packets arrive, $A_{k, j}$.

Considering a finite buffer of size, $B$, the boundary conditions for $\pi_{i, j}$ become

$$
\pi_{B, j}=\sum_{k=\max (0, B-n \cdot N+n)}^{B} \sum_{l=B+n-k}^{n \cdot N} \pi_{k, l} \cdot A_{l, j} .
$$

The equation illustrates that there are $k$ packets in the queue and $l$ new packets arrive, $\pi_{k, l}$. The $l$ newly arrived packets ensure, that even if $n$ packets are removed from the queue there are $B$ packets in the queue after the time slot where in addition $j$ new packets arrive, $A_{l, j}$.

Finally, an equation that links $\pi_{i, j}$ to the traffic load is needed. If no packets are lost we note that the total load out of the buffer is, $n \cdot N \cdot \rho / N=n \cdot \rho$. This means that in mean $n-n \cdot \rho$ wavelengths are unused corresponding to the case 
where less than $n$ packets are present in the buffer. In case of no loss, we therefore get

$$
n \cdot(1-\rho)=\sum_{i=0}^{n} \sum_{j=0}^{n-i} \pi_{i, j} \cdot(n-i-j) .
$$

The term to the right is explained as follows. Because $\pi_{i, j}$ describes the probability of having $i \leq n$ packets in the queue while $j<n$ packets arrive, $i+j$ packets need to be treated in the given time slot. Since there are $n$ wavelengths available at the output, up to $n$ packets can be removed from the queue. However, if $i+j$ is less than $n$, then $n-(i+j)$ wavelengths will be unused which happens with the probability, $\pi_{i, j}$. Thereby, the summation gives the mean number of unused wavelengths.

Using (8) we can find the probabilities, $\pi_{i, j}$, under the assumption that no packets are lost and that the queue size is limited to $B$ packets ( $B / n$ fiber delay-lines in addition to a direct connection without delay). To include packet loss in the model, a conditioned probability analysis is carried out as in [9] to find the probabilities, $\hat{\pi}_{i, j}$, for which loss is included

$$
\hat{\pi}_{i, j} \cdot F=\pi_{i, j}, \quad F=\sum_{i=0}^{B} \sum_{j=0}^{n \cdot N} \pi_{i, j} .
$$

Hereby, a complete description of the buffering process is obtained and the packet loss probability for the queuing process can be calculated.

\section{Calculation of the Packet Loss Probability}

Using the above calculated transition probabilities, the packet loss probability (PLR) can be found as 1-PSR where PSR is the packet success probability. The PSR is given as the mean number of packets that leave the queue in each time slot divided by the mean number of packets destined for the queue in each time slot, $n \cdot \rho$, giving

$$
\operatorname{PLR}=1-\mathrm{PSR}=1-\frac{n-\frac{1}{F} \sum_{i=0}^{n} \sum_{j=0}^{n-i} \pi_{i, j} \cdot(n-i-j)}{n \cdot \rho}
$$

where the nominator in the second term is the mean number of packets that leave the queue in every time slot. With this, the traffic model describing bursty traffic conditions for the general WDM packet switch in Fig. 2 is derived.

\section{MOdEL VERIFICATION}

To verify the model we compare analytic results with simulated values for a $4 \times 4$ switch with $7(B / n+1=8)$ delaylines and with a mean burst length $\beta$ of four. Simulations are carried out by using the Monte Carlo method [14] to generate the arrival processes. As seen in Fig. 7 where the PLR is shown versus the traffic load, $\rho$, of each of the $n$ wavelength channels per inlet, the results of the two methods show good agreement. We, therefore, conclude that our theoretical model can be used to analyzes the influence of TOWC's on the traffic performance. Apart from verifying the model, Fig. 7 shows

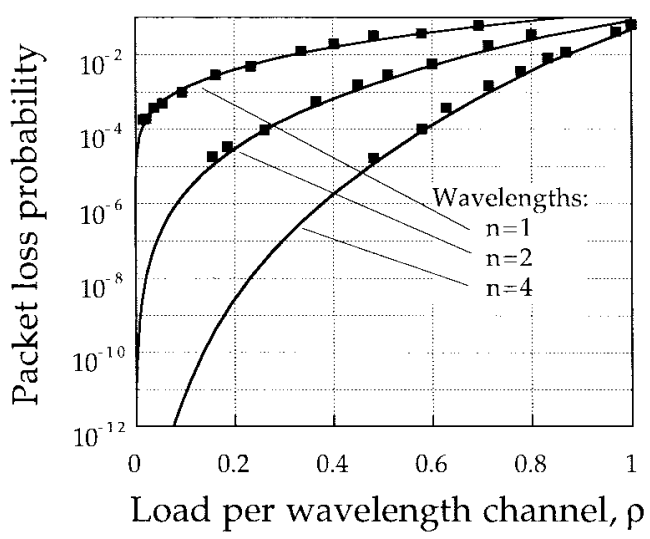

Fig. 7. Packet loss probability versus channel load for a $4 \times 4$ switch with seven delay lines. The mean burst length is four. Full curves are analytically calculated results while dots are simulated values.

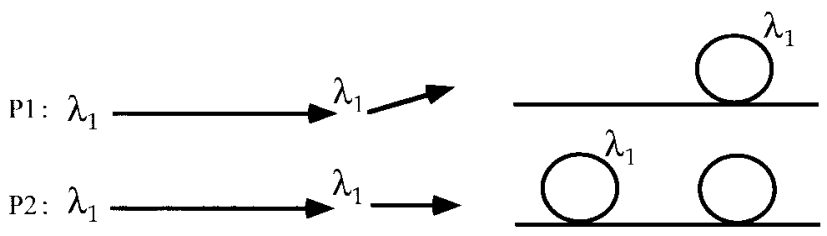

(a)

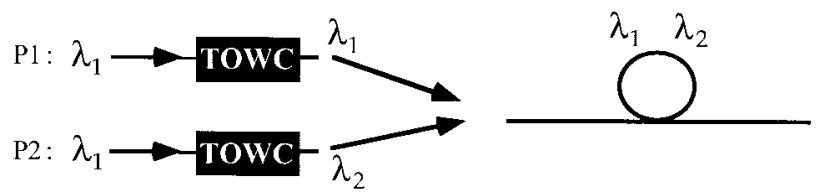

(b)

Fig. 8. Two simultaneously incoming packets, P1 and P2, destined for the same outlet have the same wavelength, $\lambda_{1}$. Without converters, two fiber delay lines are needed to store the packets, whereas with converters only one delay line is needed.

that bursty traffic has a significant influence on the acceptable loads. With uniform traffic, a load of 0.75 is acceptable (@PLR $=10^{-10}$ ) for $n=4$ [15], while for the bursty traffic situation in Fig. 7 this value is approximately 0.15. Although the bursty traffic reduces the allowed load, it is indicated that as the number of wavelengths is increased the packet loss probability decreases and thereby the impact of bursty traffic is reduced. The reason for this is the implementation of tuneable wavelength converters that allows full exploitation of the wavelength domain by addressing empty "slots" in the buffers. Fig. 8 gives a simple schematic that illustrates how TOWC's ease the problem of buffering and at the same time how wavelengths are allocated to packets. Two packets (P1, P2) arrive at the same wavelength $\lambda_{1}$ simultaneously and are destined for the same outlet. Without wavelength converters, no wavelength assignment takes place and two fiber delaylines are needed to store the packets. With tuneable wavelength converters one of the packets can be converted to another wavelength, i.e., $\lambda_{2}$, and thus only one fiber delay-line is needed. Evidently, this method can be taken further. If for example two wavelength channels are used $(n=2)$ and four packets arrive simultanously at $\lambda_{1}$ and all the packets are destined for the same outlet, then conversion of two packets to $\lambda_{2}$ is required followed by storage in two fiber delay-lines. 


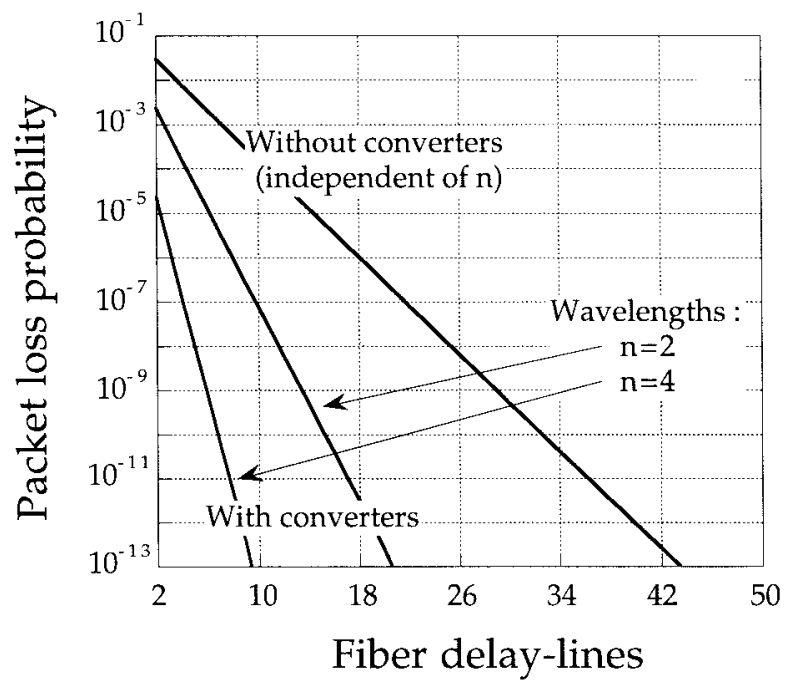

(a)

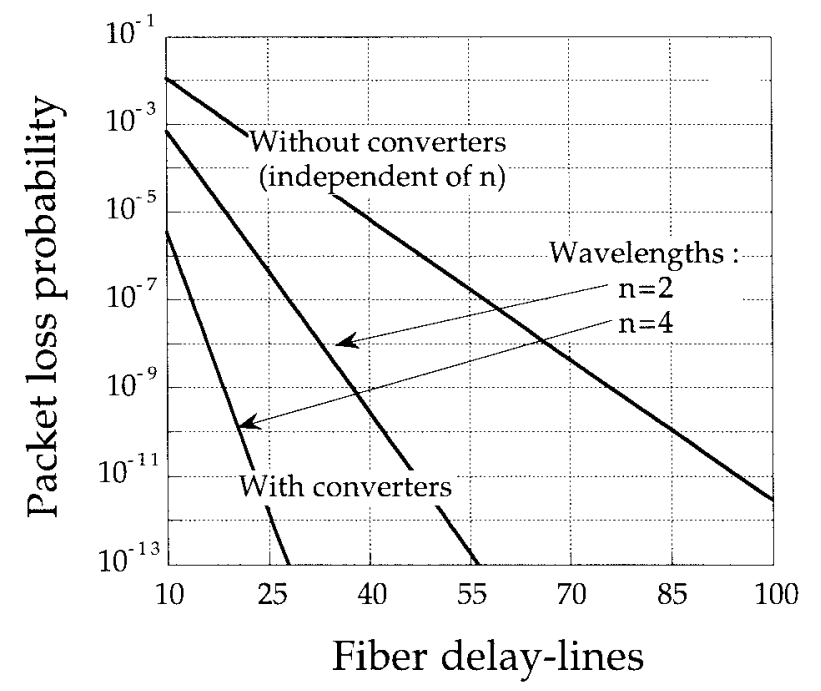

(b)

Fig. 9. Packet loss probability versus the number of fiber delay lines for a burstiness of two, $N=4$ and $n=4$ where (a) is for a load per channel of 0.4 while (b) is for a load of 0.8 .

\section{COMPLEXITY AND ThroughPUT ANALYSIS}

The reduction of the required number of fiber delay-lines due to the TOWC's is further verified in Fig. 9(a) and (b) that give the packet loss probability versus the number of fiber delay-lines for a $4 \times 4$ switch and a burstiness of 2 . Fig. 9(a) is for a channel load of $\rho=0.4$ while Fig. 9(b) is for a channel load of $\rho=0.8$. In both figures the number of wavelength channels, $n$, is the parameter and results with and without tuneable converters are shown. Evidently, the packet loss probability is independent of the number of wavelength channels when there are no converters. With converters, the packet loss probability decreases as the number of channels increase due to the buffering process described by Fig. 8. As expected, the PLR increases as the load is increased. At a channel load of $0.4,33$ delay-lines are required in a switch block without converters to obtain a PLR of $10^{-10}$. With converters and with two and four wavelength channels, 16 and 7 delay lines are required, respectively. At a channel load of 0.8

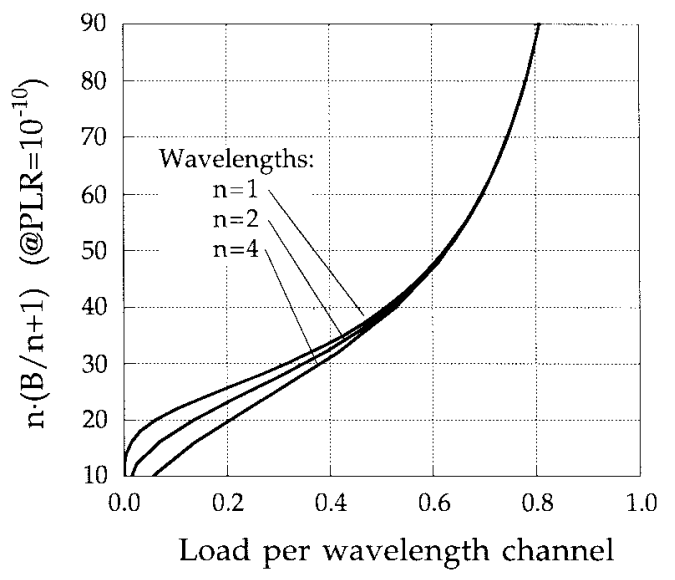

(a)

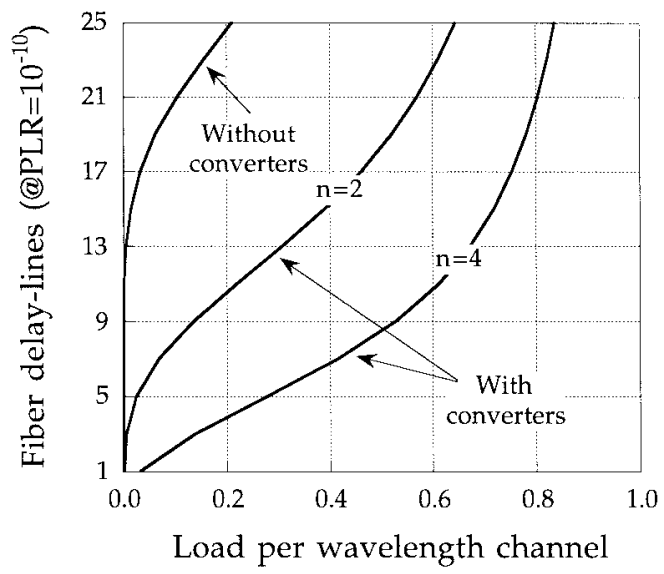

(b)

Fig. 10. Complexity (a) and required number of fiber delay-lines (b) versus the load per wavelength channel ( $@ P L R=10^{-10}$ ). The switch size is $4 \times 4$ and the parameter $n$ is the number of wavelength channels per in- and outlet. The burstiness of each channel is two.

the corresponding numbers are 86,43 , and 21 . Studying these numbers, we find that the product of the number of fibers in the buffer and the number of wavelength channels, $n \cdot(B / n+1)$, is almost constant for both channel loads. The size of the space switch of the configuration in Figs. 2 and 3 depends on this product which indicates that the space switch size remains constant when the number of wavelength channels is increased. This indication is confirmed in Fig. 10(a) and holds independent of the channel load as seen from the coinciding curves. The figure gives the mentioned product as function of the channel load (@PLR =10 ${ }^{-10}$ ) for a $4 \times 4$ switch with one, two, and four wavelength channels per in- and outlet. The actual number of delay-lines, $B / n$, is given in Fig. 10(b).

Fig. 10(a) shows that the space switch size will be constant using the wavelength converters. Thus, by increasing the number of wavelength channels the throughput of the switch ( $n \cdot N \cdot \rho$ times the channel bit rate) is increased without increasing the number of gates in the space switch. We note of course, that the component count for the total switch is not constant since for each new channel an extra TOWC is needed per inlet thus increasing the component count. On the other hand, for a fixed throughput per fiber $n \cdot \rho$ of say 0.8 the use of TOWC's together with WDM fiber delay-line buffers 


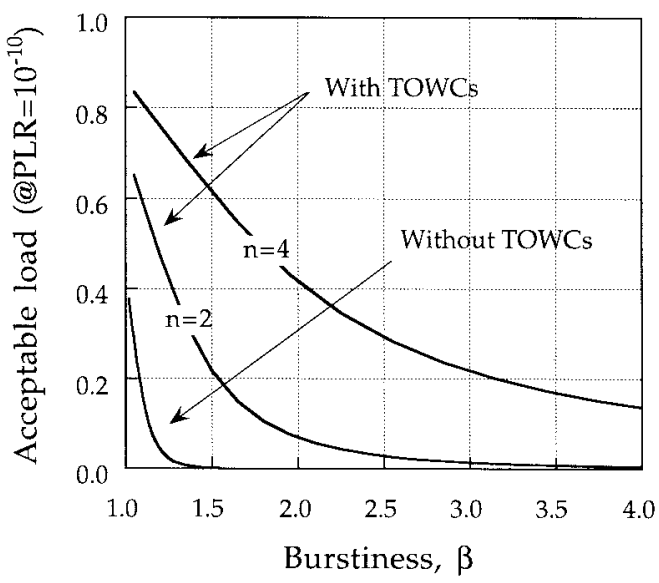

Fig. 11. Highest acceptable channel load ( @PLR $=10^{-10}$ ) versus burstiness. The switch size is $4 \times 4$ and the number of delay lines is seven $(B / n+1=8)$.

drastically decreases the product, $n \cdot(B / n+1)$, from $\sim 85$ ( $n=1$ and $\rho=0.8)$ to $\sim 15(n=4$ and $\rho=0.2)$ and thereby the number of gates in the space switch from $\sim 1360$ to $\sim 240$.

The calculations in Figs. 9 and 10 are for a burstiness of 2. If the burstiness is increased the number of fiber delaylines increases. This means, that if the number of delay-lines is kept constant the possible traffic load will decrease when the burstiness is increased. For a $4 \times 4$ switch with a fixed number of $7(B / n+1=8)$ fiber delay-lines in the buffer, Fig. 11 clarifies the important role of the TOWC's. The figure gives the maximum tolerated load per wavelength channel as function of the burstiness when a packet loss probability of $10^{-10}$ is required. Results are given with and without converters for two and four wavelength channels per in- and outlet. As expected, the burstiness degrades the obtainable switch throughput significantly. On the other hand, Fig. 11 shows that tuneable wavelength converters can be used to compensate for the impairment due to bursty traffic. Clearly, for the same number of fiber delay-lines and for increasing burstiness a much higher traffic load can be switched when TOWC's are employed. For a moderate burstiness of two and with four wavelength channels per in- and outlet, the throughput is only $160 \mathrm{Mb} / \mathrm{s}$ without tuneable converters while it is increased to $64 \mathrm{~Gb} / \mathrm{s}$ with tuneable converters assuming a channel bit rate of $10 \mathrm{~Gb} / \mathrm{s}$.

Another point to note when using wavelength converters is the higher allowed burstiness. If we return to the case of a fixed throughput per fiber $n \cdot \rho$ of 0.8 and use four wavelength channels (i.e., $n=4$ and $\rho=0.2$ ) Fig. 11 shows that the use of TOWC's increases the tolerated burstiness from 1.1 to 3.2.

\section{CONCLUSION}

A traffic model describing the traffic performance of a photonic WDM packet switch under bursty traffic patterns has been derived and verified. The model has been used to investigate the number of fiber delay-lines in the optical buffers that are required for a given traffic performance. The analysis has focused on the comparison of WDM packet switches with and without tuneable optical wavelength converters. The analysis shows that the converters highly improve the buffering process. As an example, the switch throughput for a $4 \times 4$ switch with four wavelength channels per in- and outlet and seven fiber delay lines in the buffer can be increased from 160 $\mathrm{Mb} / \mathrm{s}$ without converters to $64 \mathrm{~Gb} / \mathrm{s}$ with tuneable wavelength converters when a channel bit rate of $10 \mathrm{~Gb} / \mathrm{s}$ is assumed. Moreover, by using our analytical model we find, that for a given number of fiber delay lines in the buffer, the maximum tolerated load can be increased by increasing the number of wavelength channels per in- and outlet. Importantly, when using tuneable wavelength converters, the switch throughput can be increased by increasing the number of wavelength channels per in-and outlet without increasing the component count of the space-switch in the configuration.

\section{REFERENCES}

[1] C. Brackett, "Dense wavelength division multiplexing networks: Principles and applications," IEEE J. Select. Areas Commun., vol. 8, pp. 948-964, Aug. 1990.

[2] K. Sato, S. Okamato, and H. Hadama, "Network performance and integrity enhancement with optical path layer technologies," IEEE J. Select. Areas Commun., vol. 12, pp. 159-170, Jan. 1994.

[3] P. Green et al., "All-optical packet-switched metropolitan-area network proposal," J. Lightwave Technol., vol. 11, pp. 754-763, May 1993.

[4] A. Fioretti, F. Masetti, and M. Sotom, "Transparent routing: The enabling factor toward all-optical networking," in Proc. ECOC'94, Florence, Italy, 1994, vol. 2, pp. 503-509.

[5] K. Wünstel, M. Schilling, W. Idler, G. Eilenberger, S. Bunse, and W. Rehm, "Multidimensional optical switching with advanced key components," in Proc. ECOC'93, Montreux, Switzerland, 1993, vol. 2, pp. 89-92.

[6] S. Kuroyanagi, T. Nishi, and T. Maeda, "A photonic ATM switching system using wavelength routing cell selector and optical fiber travelingtype buffer memory," in Proc. ECOC'94, Florence, Italy, 1994, vol. 2, pp. $545-548$.

[7] J. M. Gabriagues and J. B. Jacob, "Exploitation of the wavelength domain for photonic switching in IBCN," in Proc.ECOC'91, Paris, France, 1991, vol. 2, pp. 59-66.

[8] L. Ditmann and S. B. Jacobsen, "Statistical multiplexing of identical bursty sources in an ATM network," in Proc. GLOBECOM'88, 1988, pp. 1293-1297, paper 39.6.

[9] T. Hou and A. K. Wong, "Queuing analysis for ATM switching of mixed continuous-bit-rate and bursty traffic," in Proc. INFOCOM'90, 1990, vol. 2, pp. 660-667.

[10] M. Burzio, P. Cinato, R. Finotti, P. Gambini, M. Puleo, E. Vezzoni, and L. Zuchelli, "Optical cell synchronization in an ATM optical switch," in Proc.ECOC'94, Florence, Italy, 1994, vol. 2, pp. 581-584.

[11] L. Zuchelli, M. Burzio, and P. Gambini, "New solutions for optical packet delineation and synchronization in optical packet swicthed networks," in Proc. ECOC'96, Oslo, Norway, 1996, vol. 3, pp. 3.301-3.304.

[12] A. K. Wong, "Traffic modeling and buffer dimensioning in an ATM switch with shared-memory end-grids," in Proc. ISS'92, Yokohama, Japan, Oct. 1992, vol. 1, pp. 239-243.

[13] S. Liew, "Performance of various input-buffered and output-buffered ATM switch design principles under bursty traffic: Simulation study," IEEE Trans. Commun., vol. 42, pp. 1371-1379, Feb./Mar./Apr. 1994

[14] White, Analysis of Queuing Systems, 1st ed. New York: Academic, 1975.

[15] S. L. Danielsen, B. Mikkelsen, C. Jorgensen, T. Durhuus, and K. E. Stubkjaer, "WDM packet switch architectures and analysis of the influence of tuneable wavelength converters on the performance," $J$. Lightwave Technol., vol. 15, pp. 219-227, Feb. 1997.

Soren L. Danielson was born in Copenhagen, Denmark, in 1970. He received the M.Sc. degree in electronic engineering from the Technical University of Denmark, Copenhagen, in 1994 and the Ph.D. degree in optical communication in 1997 from the Technical University of Denmark.

His field of interest is high-capacity optical networks with emphasis on traffic analysis and switch blocks including optical amplifiers as functional elements. 
Carsten Joergensen was born in 1967. He received the M.Sc. degree in electronic engineering in 1991 and the Ph.D. degree in optical communications in 1997 from the Technical University of Denmark, Copenhagen.

$\mathrm{He}$ is now with Ericsson, Denmark.

Benny Mikkelsen was born in 1970 and received the M.Sc. and Ph.D. degrees from the Technical University of Denmark (TUD), Copenhagen, Denmark, in 1987 and 1995, respectively.

He is currently with Lucent Technologies, Holmdel, NJ.
Kristian E. Stubkjaer (S'80-M'81) was born in Denmark in 1953. He received the M.Sc. and Ph.D. degrees from the Technical University of Denmark, Copenhagen, in 1977 and 1981, respectively. From 1979 to 1981, he studied at the Tokyo Institute of Technology, Tokyo, Japan, with a scholarship from the Japanese Government.

From 1981 to 1982 , he was drafted for military service at the Danish Defence Research Establishment, Copenhagen. From 1982 to 1983, he was a Visiting Scientist at the IBM Thomas J. Watson Research Center, Yorktown Heights, NY. From 1985 to 1990, he was Director of the Electromagnetics Institute and currently he is serving as Chairman for the Electrotechnical Committee under the Technical Research Council (Danish Ministry for Education). He is now an Associate Professor at the Electromagnetics Institute, Technical University of Denmark, where he is working in the field of optical communication. 